ORIGINAL ARTICLES

\title{
Phase plane quantification of single-joint smoothness
}

\author{
Michael Wininger*1,5,6, Alex Krasner², Nam Hun Kim³ ${ }^{3}$ William Craelius 3 ,4 \\ ${ }^{1}$ Department of Rehabilitation Sciences, University of Hartford, United States \\ ${ }^{2}$ Stanford Research Institution, Princeton, NJ, United States \\ ${ }^{3}$ Department of Biomedical Engineering, Rutgers University, United States \\ ${ }^{4}$ Sichuan Provincial Rehabilitation Hospital affiliated with Chongqing Medical University, Sichuan Bayi Rehabilitation Center, \\ China \\ ${ }^{5}$ Cooperative Studies Program, Department of Veterans Affairs, United States \\ ${ }^{6}$ Department of Biostatistics, Yale University, United States
}

Received: March 29, 2018

DOI: $10.5430 /$ jbei.v4n1p40
Accepted: May 4, 2018

URL: https://doi.org/10.5430/jbei.v4n1p40

\begin{abstract}
We report on a metric of single joint movement smoothness based on phase plane analysis of trajectories of the wrist about the elbow. Overall smoothness was quantified as the Phase Area Ratio (PAR), comparing the total area circumscribed by the acceleration-velocity (A-V) curve, to the area of its convex hull; PAR ranges from 0 (perfectly smooth) to 1 (gross motor impairment). Elbow flexion records obtained from a cohort study showed that PAR was significantly different in intact $\left(P A R=9.4 \times 10^{-4} \pm 6.6 \times 10^{-4}\right.$, group average, $\left.N=18\right)$ versus chronic stroke patients $(0.11 \pm 0.15, N=9$; Wilcoxon rank-sum on group means: $P<.0001)$. Separate simulations showed that PAR was appropriately insensitive to velocity asymmetry and to scale factors, e.g. range of motion, peak- and average velocity, and movement duration. We conclude that PAR is an attractive smoothness measure, as it accomplishes four objectives: 1) insensitivity to scale factors unrelated to trajectory shape, 2) discrimination of an intact versus impaired cohort, 3) reporting a near-zero impairment for healthy actors, responding appropriately to asymmetries commonly observed in human movement, and 4) operation on a fixed, closed scale.
\end{abstract}

Key Words: Acceleration, Jerk, Kinematics, Phase plane, Rehabilitation, Motor control, Stroke, Reaching, upper-limb

\section{INTRODUCTION}

Smoothness is a hallmark of healthy neuromotor control, and its absence is diagnostic of neuromuscular pathologies that accompany hemiparesis, including spasticity and hyperkinesis. Conceptually, smoothness is an intuitive and easily identifiable trait, but its quantification is a complex enterprise, with no single measure yielding consistent, interpretable results.

The current approach to quantifying smoothness predicates on a hypothetical neuromotor strategy that attempts to opti- mize a singular analytical function: jerk, the summed rate of accelerative changes of during a movement. ${ }^{[1]}$ Thus jerk quantifies smoothness according to the degree which the movement minimizes its accelerative transients. The problem with this classification is that, given a set of initial conditions, only a single trajectory can meet it. Instead, we know many different motion trajectories can efficiently achieve the same target. This fact is illustrated most clearly by skilled actors such as blacksmiths, working their hammer precisely to the anvil using a different trajectory for each cycle. ${ }^{[2]}$ Moreover, goal-directed movements in general are asymmetric in the ac-

\footnotetext{
*Correspondence: Michael Wininger; Email: wininger@ hartford.edu; Address: Department of Rehabilitation Sciences, University of Hartford, 200 Bloomfield Avenue, West Hartford, CT 06117, United States.
} 
celeration and deceleration phases. ${ }^{[3]}$ Jerk classifies many of these visually smooth and accurate movements as un-smooth. Perhaps because of this flaw, the jerk metric has reported anomalous increases following intensive rehabilitation, ${ }^{[4,5]}$ and has failed to identify performance deficits in clinical populations with clear motor impairment. ${ }^{[6,7]}$ How do we resolve these contradictions?

Here we define a smooth movement as one which transcribes a trajectory consisting of a biphasic velocity profile with a sigmoidal acceleration phase followed by a sigmoidal deceleration phase. The definition specifically does not assume a symmetric bell-shaped velocity profile, since this idealized model is rarely executed. ${ }^{[3,8,9]}$ Here, we propose a robust measure of motion smoothness that meets the following requirements: 1) complete insensitivity to scale-factors including range of motion, duration, and peak- or average velocity, 2) consistent and interpretable baseline values for motions of healthy subjects, 3 ) ability to discriminate healthy from impaired motions, and 4) a scale from zero to one, with zero representing ideal or completely proficient performance and one representing complete impairment. Such properties are prerequisite to reliable comparisons across diverse populations.

Where kinematic analyses in the temporal domain data require corrections for bias due to scale factors, ${ }^{[10,11]}$ transforming kinematic data into the acceleration-versus-velocity (A-V) phase plane using a ratio-metric approach, avoids this problem entirely. Specifically, our solution is the phase area ratio (PAR), which compares the phase area occupied by the trajectory against an ideal convex polygon, a minimally fit convex hull. Completely smooth movements as defined above, will produce phase portraits identical to their hulls, whereas non-smooth movements would deform the convex hull to varying degrees. In the case of uni-phasic acceleration and deceleration and zero accelerative transients, $\mathrm{PAR}=0$; PAR will increase towards unity as accelerative transients grow. Here, we test PAR on both simulated data and real data from human subjects. We show that PAR meets all four requirements for a valid smoothness metric, offering an accurate and versatile metric of motor proficiency.

\section{Methods}

\subsection{Phase area ratio}

The PAR is calculated as follows: first, position versus time data are converted into the acceleration-versus-time phase space. In the A-V phase plane, smooth traces yield a wholly convex polygon; symmetric velocity data yield an elliptical portrait (Figure 1 Left panels). While asymmetrical velocities yield irregular-but still strictly convex-pseudoellipses (Figure 1 Center panels); data containing accelerative transients create local deviations from the ellipse, yielding two features not seen in the portraits of smooth movements: 1) self-intersecting loops, and 2) bulging prominences. We take these disruptions to the polygonal complexity of the phase portrait to represent degrees of improficiency.
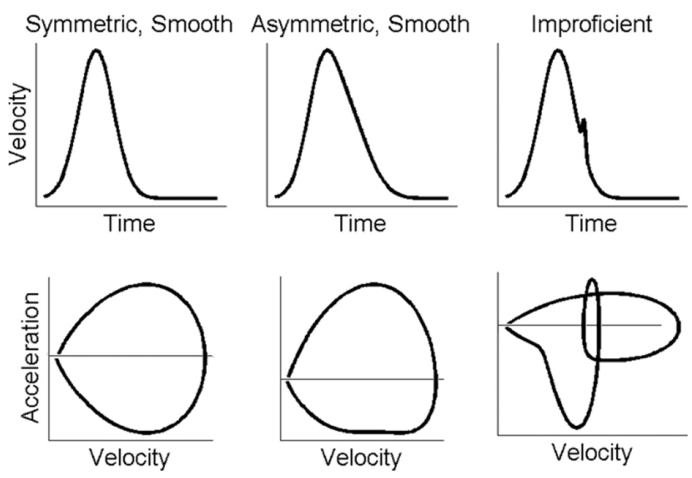

Figure 1. Phase-plane portrayals of three simulated flexions: proficient, asymmetric, and improficient. Left panels show a completely proficient flexion (top), and its phase portrait (bottom), which is smoothly convex and symmetrical. Center panels show an asymmetric but smooth flexion (top), and phase portrait (bottom) which is distorted, but remains convex. Right panels show an improficient flexion with transient second peak in velocity, yielding it's a distorted phase portrait with multiple convex or overlapping traces.

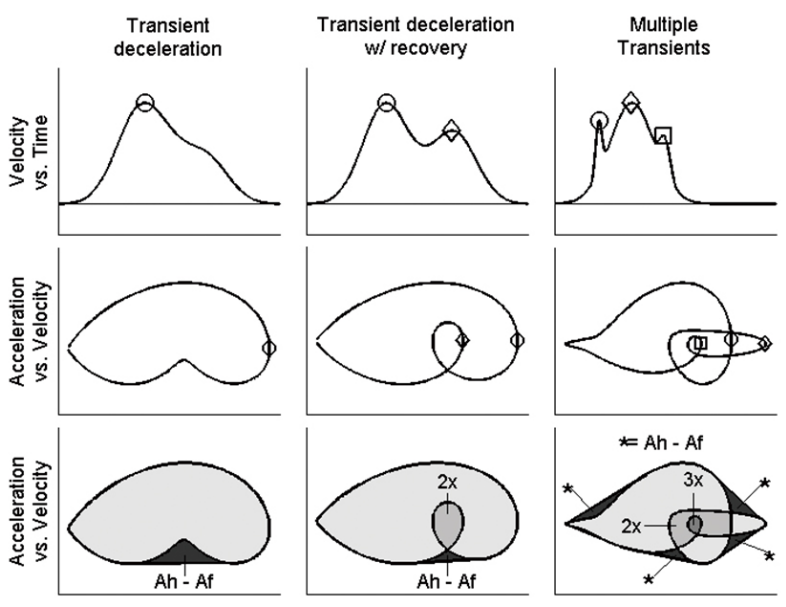

Figure 2. Depiction of PAR calculation for 3 simulated trajectories. Left panels show a transient deceleration with modest concavity in phase portrait. Center panels show a two-peaked velocity profile with concavity and overlapping region; the overlap area contributes doubly to the phase area. Right panels show a highly irregular velocity portrait with multiple convex features and overlaps; overlaps contribute doubly- or triply to total phase area, as appropriate. $\mathrm{Ah}=$ Hull area, the region encompassed by the convex hull fitted around the entire phase portrait. Af = Footprint area, the region enclosed by the perimeter of the phase portrait. 
PAR is a single number that quantifies the motor behavior illustrated by these phase portraits. Calculations involve measuring three features: area of the "footprint" of the phase portrait (Af), area of the convex hull enclosing the phase portrait (Ah), and the area of any additional overlaps within the phase plot created by the self-intersecting loops (Al), as depicted in Figure 2.

We define the PAR as $1-(A f \div(A h+A l))$. In the case of no accelerative transients and monophasic acceleration and deceleration, the phase plane is a wholly convex polygon, $A f=A h, A l=0$, and $P A R=0$; as accelerative transients grow, $A h-A f>0, A l>0$ and $P A R>0$.

\subsection{Human subjects data collection}

Subject testing was approved by the Rutgers University Institutional Review Board. Subjects were introduced to the study methods and goals by one of the authors, and were entered into the study after they read, consented and signed the approved informed consent. Eighteen healthy controls (10 male, 8 female; age range 20-64) and nine subjects with stroke were studied (6 male, 3 female, age range 41-79 years, and time post-CVA ranged from 5 to 72 months). Clinical documentation and testing were done at Rutgers University and the Kessler Institute for Rehabilitation (Edison, NJ). The patient cohort comprised a mix of right-affected and leftaffected subjects, and only their affected arm was tested; all control subjects were right-dominant and tested on the right side. Subjects were seated with their arms supported against gravity and comfortably held with Velcro ${ }^{\mathrm{TM}}$ by the Mechanical Arm Supporter and Tracker (MAST), which restricts movement about the shoulder and wrist, allowing isolated single-joint articulation of the forearm about the elbow. ${ }^{[7]}$ All participants were instructed to flex and extend their elbow at a comfortable pace and to a comfortable range; there were not temporal or spatial targets to achieve.

\subsection{Signal processing}

The MAST was equipped with a potentiometric goniometer, located on axis with the elbow, to register elbow position and an accelerometer (ADXL330) located near the wrist. Signals were sampled at $40 \mathrm{~Hz}$, bi-directionally filtered with a low-pass Butterworth filter (4 Hz cutoff), and differentiated with a point-wise difference. To reduce noise generated by differentiation, filtering was applied after both the first and second differentiation; this was done serially so as to allow for obtaining a low-pass filtered trace for each differentiation, i.e. a low-pass filtered position, a low-pass filtered velocity, and a low-pass filtered acceleration trace. Flexion cycles were segmented from the goniometric data stream by automatic extraction of local angular extrema. Since fixed criteria could not reliably detect the angular extrema in impaired subjects who often stalled within a traverse, motion endpoint was defined as a stop followed by a sustained reversal, and identified visually. Individual flexions and extensions were thus segmented from the angular position data and further analyzed.

\subsection{Cohort study}

The most fundamental test for a performance measure is its ability to discriminate an impaired cohort from an otherwise comparable group comprised of neurologically intact persons. Additionally, it is equally important that a valid performance measure should yield consistently good scores for reasonable movements performed by healthy actors. Our objectives in this study are, firstly, to test whether PAR yields scores approaching zero for the movements performed by healthy actors, and secondly, to test whether PAR scores observed among the cohort of stroke patients are significantly greater than those in the control group. We thus devised the following hypotheses: H1: individual PAR averages $<0.01$ for each healthy subject, and $\mathrm{H} 2$ : that a statistically significant difference would be observed between the group-averages of PAR scores between the two groups, at $P<.05$.

\subsection{Simulations}

\subsubsection{Velocity asymmetry}

In order to test PAR performance in the case of asymmetry, velocity profiles of various symmetries were simulated via a Fisk log-logistic probability distribution function, given by

$$
f(t, a, b)=\frac{b}{a} \cdot\left(\frac{t}{a}\right)^{b-1} \cdot\left(1+\left(\frac{t}{a}\right)^{b}\right)^{-2}
$$

where $t$ is time (here set to $1 \leq t \leq 500$ in increments of $0.5), 100 \leq a \leq 250$ is a breadth parameter, and $3 \leq b \leq 20$ is a parameter that determines length of the distribution tail. Skewness for each simulated velocity profile was calculated via the standard definition

$$
\gamma=\frac{\frac{1}{n} \sum_{i=1}^{n}\left(x_{i}-\bar{x}\right)^{3}}{\left(\sqrt{\frac{1}{n} \sum_{i=1}^{n}\left(x_{i}-\bar{x}\right)^{2}}\right)^{3}}
$$

where $\gamma=0$ is perfectly symmetrical, $\gamma<0$ indicates a large tail before the peak, and $\gamma>0$ indicates a large tail after the peak.

We systematically compared the performance of PAR with previously used smoothness measures: 1) nondimensionalized jerk, and 2) phase jerk. Both jerk measures are based on the standard definition of smoothness as the 
minimization of the integral of the square of the first time derivative of acceleration, but non-dimensionalized jerk predicates on the conventional velocity-versus-time trace

$$
\eta=\frac{T^{5}}{\mathrm{~A}^{2}} \int_{0}^{T}\left[\frac{d^{3}}{d t^{3}} \theta(t)\right]^{2} d t
$$

where $A=$ movement amplitude and $T=$ total time (duration of movement) (Takada et al., 2006), and phase jerk

$$
\varphi=\frac{1}{\max \left(\dot{\theta}\left(\theta^{*}\right)\right)} \int_{\theta_{i}^{*}=0}^{\theta_{i}^{*}=1}\left[\frac{d^{2}}{d \theta^{2}} \dot{\theta}\left(\theta^{*}\right)\right]^{2} d \theta^{*}
$$

Where $\dot{\theta}\left(\theta^{*}\right)$ is angular velocity $\dot{\theta}=d \theta(t) / d t$ as a function of normalized position $0 \leq \theta^{*} \leq 1$. $^{[11]}$ We note that by design, $\varphi$ is the scale-independent phase-plane analog of $\eta$, and therefore the closest comparator between PAR and jerk-derived measures based in the time domain. Here, our objective was to test whether PAR would be insensitive to even moderate asymmetry in otherwise smooth movements (i.e. free of accelerative transients); our operating hypothesis was expressed as H3: PAR would remain below the $P<.01$ threshold for all movements, regardless of their skewness.

\subsubsection{Scale factors}

We tested PAR for its possible dependency on range of motion (ROM) or duration, similarly to a previous study of jerk based measures. ${ }^{[12]}$ To make this comparison, PAR was computed for 12 repetitions each of both simulated and human movement cycles on original ROM and following three decimations (to $0.1 \times \mathrm{A}, 0.01 \times \mathrm{A}$, and $0.001 \times \mathrm{A}$, where $\mathrm{A}$ is amplitude of $\mathrm{ROM}$ ), and at three different durations (original sampling rate and down-sampled to one-half and one-quarter sampling rate). Our objective was to test whether PAR was sensitive to scale factors unrelated to trajectory shape; our hypothesis was $\mathrm{H} 4$ : variance in PAR across all permutations would yield less than $1 \%$ change from the true observed PAR score in the un-perturbed record.

\section{RESUlts}

\subsection{Cohort study}

In total, 688 movement records were observed from 27 subjects. The movements observed in our subjects would be considered "typical" of any cohort study, with descriptive statistics within the expected range: $91.8 \pm 16.2^{\circ}$ versus $72.3 \pm 25.1^{\circ} \mathrm{ROM}$ (healthy versus stroke), $58.8 \pm 7.3^{\circ}$ versus $37.6 \pm 11.9^{\circ} / \mathrm{s}$ peak velocity, $33.0 \pm 4.7^{\circ}$ versus 15.6 $\pm 8.9^{\circ} / \mathrm{s}$ average velocity, and $2.7 \pm 0.9 \mathrm{~s}$ versus $8.8 \pm 7.4 \mathrm{~s}$ movement duration. Examples of trajectories are shown in Figure 3.
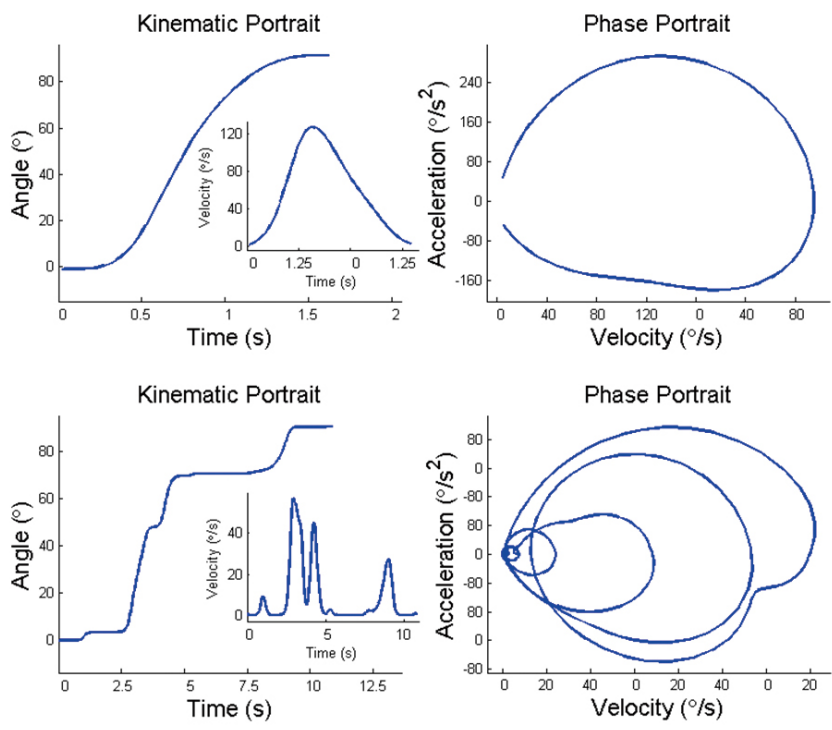

Figure 3. Sample flexion trajectories from unimpaired subject (Top) and subject with chronic stroke (Bottom). The left panels show kinematic portraits of position versus time, and velocity versus time (Left Insets). Note the smooth sigmoidal angle versus time curve, and (inset) the smooth, moderately asymmetric bell-shaped velocity profile. The right panels show the corresponding phase portraits of acceleration versus velocity. Note the relatively smooth hull for the control subject in contrast to the distorted profile and multiple inner loops in the phase portrait of the stroke subject.

The within-subject PAR scores for healthy subjects was uniformly low: Range 0.00012 to 0.00243 ; PAR for the chronic stroke patients was modest to moderate: Range 0.01247 to 0.46493 . PAR was significantly different in intact $\left(P A R=9.4 \times 10^{-4} \pm 6.6 \times 10^{-4}\right.$, group average, $N=18)$ versus chronic stroke patients $(0.11 \pm 0.15, N=9)$; Wilcoxon-rank sum on group means: $P<.0001$. Thus, H1 and $\mathrm{H} 2$ were confirmed: PAR yields uniformly low $<0.01$ score for healthy actors, and significantly higher score for impaired subjects.

\subsection{Asymmetry}

A skewed probability distribution was employed to simulate velocity data of variable symmetries. The range of skewness data was large: $-1.4 \leq \gamma \leq 1.4$, and as expected, all three measures, PAR, non-dimensionalized jerk $\eta$, and phase jerk $\varphi$, responded equally whether the skew was positive or negative (i.e. the outcomes were reflected about the $\gamma=$ 0 axis). For all data, PAR yielded a low score well below the pre-defined threshold for healthy movements; we thus confirm H3.

As context, we report that the two jerk-derived measures re- 
port very large scores for these data, and -counterintuitivelylarger scores for symmetric movements than for asymmetric movements.
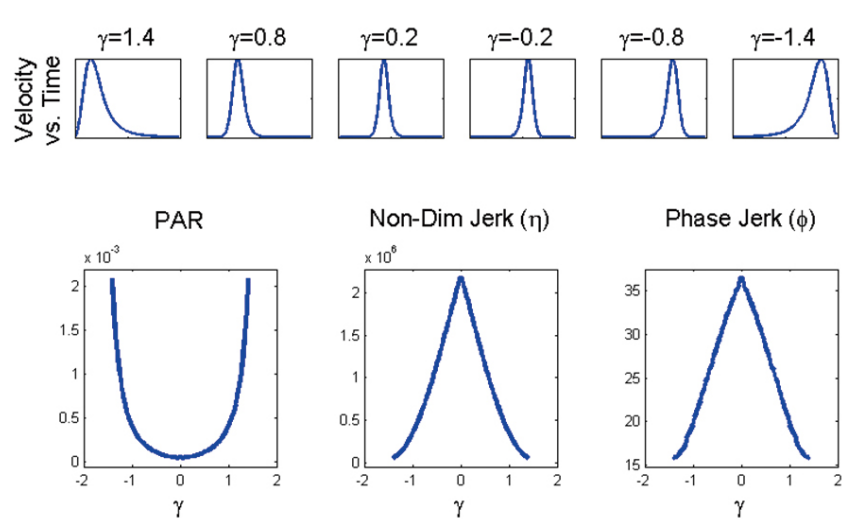

Figure 4. PAR yields nearly perfect scores (PAR $\ll 0.01$ ) for all traces, regardless of symmetry. Top row shows snapshots of the simulated velocity traces, viz. Equation 1, across a range of symmetries; Bottom row shows response of three smoothness measures: PAR, non-dimensionalized jerk, and phase jerk; only PAR shows an appropriate decrease with symmetry, and all values are well below the PAR $<0.01$ threshold for high proficiency.

\subsection{Scale factors}

PAR was tested with varying scale factors: amplitudes and durations of movement, and their features, and peak and average velocities. With amplitude variations, PAR maintained 6-digit precision across all amplitudes of both human and simulated data. Variations of duration, however, introduced large (orders of magnitude) changes in PAR from human subject data, but no changes in simulated data.

As a possible explanation for the apparent inconsistency between simulated and real data, we posited that the inherent irregularities in empirical as opposed to simulated data, made re-sampling an unsuitable basis for testing PAR consistency. To test this, we up-sampled the raw position-versus-time human data through interpolation to twice its original sampling density, then down-sampled back to its original length, twice differentiated both datasets (both raw, and the up-thendown sampled data), and correlated both sets (representing acceleration). The correlations were in general very low (0.5$0.6)$, meaning that the acceleration traces of observational data looked very dissimilar after re-sampling. By contrast, the simulated data were little changed (correlation typically greater than 0.99). Here we draw the specific conclusion that PAR's sensitivity to movement duration cannot be reasonably tested via re-sampling of observational data, but that the testing via re-sampling of simulated data is appropriate. More generally, we conclude that PAR is completely independent of scale factors, and thus, H4 is confirmed.

\section{Discussion}

\subsection{Significance}

It has long been established that measures of human motor performance are sensitive to and can be confounded by asymmetries in the velocity profile. ${ }^{[9]}$ Measures such as jerk, that operate strictly in the temporal domain, are highly sensitive to motion asymmetry, and thus are unreliable metrics. For example, jerk analysis of arm motions in subjects with Parkinson's disease, do not always report significant impairment, despite the obvious impairment and large asymmetry of movements. ${ }^{[13]}$

PAR represents a paradigm shift for standardizing movement performance analytics and more reliable and straightforward assessment of motor proficiency.

\subsection{Implications}

PAR has four useful features: (1) sensitivity only to the shape of the motion and insensitivity to scale factors, (2) reliable discrimination between patient and control cohorts, (3) consistent and interpretable baseline values for healthy subjects, and (4) performance rating on a bounded scale of 0 to 1 . While the first two features are not novel, the third and fourth are not only novel, but potentially transformative. The jerk coefficients, $\eta$ and $\varphi$, have a "bottom" but no "top", meaning that an ideal performance could -in principle- yield a score of 0 (notwithstanding potentially unrealistic assumptions about velocity symmetry), but can reach arbitrarily large values (see Figure 4). Too often, integrated measures are normalized or re-dimensionalized, limiting their ability to be compared across studies. PAR is unit-less, dimension-less, and scale-free; healthy actors yield scores uniformly very near zero (PAR < 0.01). These are attractive features that cannot be replicated by integrated measures. Thus PAR values are universal, and can be immediately compared across all platforms and populations.

From a broader perspective, PAR is agnostic with respect to neuromotor organization: Performance measures tend to intertwine with specific theories of motor planning. For instance, jerk metrics are inextricably linked to the principle that a motor plans seek to minimize them. While it is beyond the scope of this article to discuss any theories of motor planning, we note that there are many competing theories, each of which yields its own optimization-based measure. PAR works backwards from this paradigm, looking purely at the trajectory shape, and making no assumptions about the underlying motor plan. 


\subsection{Limitations}

The concept that the PAR range of zero to one spans the clinical range of complete proficiency to complete impairment was not proven. For our cohort of 18 healthy subjects, PAR ranged from 0.00012 to 0.00243 . The question of whether zero PAR can be humanly achieved is unanswered, but is probably not important to the utility of the measure. Since zero is a very hard number to achieve in any measure, we arbitrarily set a benchmark test for complete proficiency at an arbitrarily close to zero, $\mathrm{PAR}=0.01$, which was achieved by our healthy cohort. Stroke cohort PAR values ranged from 0.01247 to 0.46493 , which translates to impairment levels ranging from slightly worse than healthy to about half fully-impaired. These outcomes, i.e. statistical significance in the presence of a high standard, validates PAR, and more definitive categorization of impairments by PAR can be documented with further testing on larger populations.

\subsection{Implementing PAR}

While there are methods for calculating convex hull in large dimensional datasets, ${ }^{[14,15]}$ most commercially available computational software packages only support hull finding in the planar case (i.e. the two-dimensional A-V plane for single-joint motion). However, with regard to the computation of PAR reported here, we executed our calculations using Octave, a freeware computational environment with highly similar design and syntax to Matlab; ${ }^{[16-18]}$ these computa- tions could have also been performed using $\mathrm{R}$ or a lower-level language. Thus, for at least the computationally tractable scenario of single-joint motion, this computation can be made without the purchase of expensive proprietary software or toolboxes: PAR is an accessible measure.

\subsection{Future work}

As technology advances our ability to record human motions in more natural conditions and with finer resolutions, the need for better biomechanical metrics is increasing. For example, the Computer Assisted Rehabilitation Environment (CAREN) system can record motions and forces from an almost unlimited number of sites on the freely moving body at high temporal and spatial resolution, yielding over 1 million data points per session. An initial use of this technology has been to assess jerk and asymmetry of arm motions during walking by persons with Parkinson's disease. ${ }^{[13]}$ Phase-plane analysis offers a more efficient and standardized means to reduce these voluminous data into meaningful and accurate measures, and holds great potential as a bioinformatic approach to clinical diagnoses.

\section{ACKNOWLEDgements}

The authors thank Todd Alter and Don Yungher for their helpful contributions, and gratefully acknowledge support from the New Jersey Commission on Brain Injury Research (CBIR1R13RG023) and the Sichuan Bayi Rehabilitation Center, China.

\section{REFERENCES}

[1] Hogan N, Sternad D. On rhythmic and discrete movements: reflections, definitions and implications for motor control. Experimental Brain Research. 2007; 181(1): 13-30. PMid:17530234. https://doi.org/10.1007/s00221-007-0899-y

[2] Bernstein A. The Coordination and Regulation of Movements. Oxford Pergamon. 1967: 77-92.

[3] Morasso P. Spatial control of arm movements. Exp Brain Res. 1981; 42(2): 223-7. PMid:7262217.

[4] Goldvasser D, McGibbon CA, Krebs DE. High curvature and jerk analyses of arm ataxia. Biol Cybern. 2001; 84(2): 85-90. PMid:11205353. https://doi.org/10.1007/s004220000201

[5] Rohrer B, et al. Movement smoothness changes during stroke recovery. Journal of Neuroscience. 2002; 22(18): 8297-8304. https : //doi.org/10.1523/JNEUROSCI.22-18-08297. 2002

[6] Cozens JA, Bhakta BB. Measuring movement irregularity in the upper motor neurone syndrome using normalised average rectified jerk. J Electromyogr Kinesiol. 2003; 13(1): 73-81. https: //doi.org/10.1016/S1050-6411(02)00070-6

[7] Wininger M, Kim NH, Craelius W. Spatial resolution of spontaneous accelerations in reaching tasks. J Biomech. 2009; 42(1): 29-34. PMid:19062017. https://doi.org/10.1016/j.jbiomech. 200 8.10 .015

Published by Sciedu Press
[8] Abend W, Bizzi E, Morasso P. Human arm trajectory formation. Brain. 1982; 105(Pt 2): 331-48. PMid:7082993. Available from: ht tp://www.ncbi.nlm.nih.gov/entrez/query.fcgi?cmd=R etrieve\&db=PubMed\&dopt $=$ Citation\&list_uids $=7082993$

[9] Hogan N. An organizing principle for a class of voluntary movements. J Neurosci. 1984; 4(11): 2745-54. https://doi.org/10.1523/ JNEUROSCI . 04-11-02745.1984

[10] Wininger M. Decomposition and metrical analysis of single-joint movement of the hemiparetic upper-limb, in Biomedical Engineering. 2009, Rutgers University: New Brunswick.

[11] Wininger M. A generalized strategy for measuring performance in the velocity-position phase plane Journal of Motor Behavior. 2012.

[12] Wininger M, Kim NH, Craelius W. Reformulation in the phase plane enhances smoothness rater accuracy in stroke. Journal of Motor Behavior. 2012; 44(3): 149-159. PMid:22420840. https: //doi.org/10.1080/00222895.2012.663012

[13] Sternad D, Turvey MT, Schmidt RC. AVERAGE PHASE DIFFERENCE THEORY AND 1/1 PHASE ENTRAINMENT IN INTERLIMB COORDINATION. Biological Cybernetics. 1992; 67(3): 223 231. https://doi.org/10.1007/BF00204395

[14] Chan TM. Optimal output-sensitive convex hull algorithms in two and three dimensions. Discrete \& Computational Geometry. 1996; 16(4): 361-368. https://doi.org/10.1007/BF02712873 
[15] Barber CB, Dobkin DP, Huhdanpaa H. The quickhull algorithm for convex hulls. ACM Transactions on Mathematical Software. 1996; 22(4): 469-483. https : //doi .org/10.1145/235815. 235821

[16] Silva I, Moody GB. An open-source toolbox for analysing and processing PhysioNet databases in MATLAB and Octave. Journal of Open Research Software. 2014; 2(1): e27. PMid:26525081.
[17] Milano F. An open source power system analysis toolbox. IEEE Transactions on Power Systems. 2005; 20(3): 1199-1206. https : //doi.org/10.1109/TPWRS. 2005.851911

[18] Schlogl A. A comparison of multivariate autogregressive estimators. Signal Processing. 2006; 86(9): 2426-2429. https ://doi.org/10 $.1016 / j$.sigpro. 2005.11.007 